\title{
USE OF SMART ANTENNAS IN AD HOC NETWORKS
}

\author{
Mohammed Ali Hussain ${ }^{1}$, P.Suresh Varma ${ }^{2}$, K. Satya Rajesh ${ }^{3}$, Hussain Basha \\ Pathan $^{4}$, Leela Madhav Sarraju ${ }^{5}$ \\ ${ }^{1}$ Assoc. Professor \& Head, Dept.of Computer Science \& Engineering, Sri Sai Madhavi \\ Institute of Science \& Technology, Rajahmundry, A.P., India. \\ hussain_ma2k@yahoo.co.in \\ ${ }^{2}$ Professor, Dept. of Computer Science \& Engineering, Adikavi Nannaya University, \\ Rajahmundry, A.P., India. vermaps@yahoo.com \\ ${ }^{3}$ Research Scholar, Dept.of Computer Science, Rayalaseema University, Kurnool, A.P., \\ India. ksatyarajeshegmail.com \\ ${ }^{4}$ Research Scholar, Dept.of Computer Science, Rayalaseema University, Kurnool, A.P., \\ India. phussain786@gmail.com \\ ${ }^{5}$ Research Scholar, Dept.of Computer Science, Dravidian University, Kuppam, A.P., \\ India. sarraju@gmail.com
}

\begin{abstract}
The capacity of ad hoc networks can be severely limited due to interference constraints. One way of using improving the overall capacity of ad hoc networks is by the use of smart antennas. Smart antennas allow the energy to be transmitted or received in a particular direction as opposed to disseminating energy in all directions. This helps in achieving significant spatial re-use and thereby increasing the capacity of the network. However, the use of smart antennas presents significant challenges at the higher layers of the protocol stack. In particular, the medium access control and the routing layers will have to be modified and made aware of the presence of such antennas in order to exploit their use. In this paper we examine the various challenges that arise when deploying such antennas in ad hoc networks and the solutions proposed thus far in order to overcome them. The current state of the art seems to suggest that the deployment of such antennas can have a tremendous impact in terms of increasing the capacity of ad hoc networks.
\end{abstract}

\section{KEYWORDS}

Directional Antennas, Medium Access Control, Routing.

\section{INTRODUCTION}

The use of smart antennas in cellular networks has increased capacity by reducing interference and enabling spatial reuse of spectrum. Typically these antennas are deployed at base-stations in these networks to sectorize cells and focus transmissions in certain directions [1] [2]. A smart antenna usually consists of an array of antenna elements that work together in order to either focus the transmitted energy in a particular desired direction or to provide uncorrelated receptions of signals that can then be combined by complex signal processing techniques to improve the received signal quality or both. The spacing between the antenna elements is on the order of the wavelength of the carrier used for communications. Consequently, as DOI : $10.5121 /$ ijcsit.2010.2605 
technology makes the use of higher frequencies feasible, the spacing between the antenna elements can be much smaller. As an example, if the elements of the antenna array were to be arranged in a cylindrical layout, the radius of the cylindrical array would be just 3.3 centimeters if the ISM 5.8 GHz band were used [3]. Similarly, if we were to use the $24 \mathrm{GHz}$ band, the radius of such a cylindrical array needs to be just around 0.8 centimeters. This in turn allows the use of small antenna elements that can be housed on mobile terminals. One could now potentially use these antennas in ad hoc networks that simply consist of mobile devices without a fixed supporting infrastructure.

The use of these antennas in mobile ad hoc networks however raises a new set of challenges. Traditional protocols (medium access control, routing and transport in particular) do not take advantage of the existence of the underlying antennas, Furthermore, in order to use the antennas effectively, support from the higher layer protocols is necessary. There has been a lot of recent interest in the design of new protocols to facilitate the use of smart antennas in ad hoc networks.

In this paper, we review the current state of the art protocols at the medium access control and discuss why they are appropriate for use with smart antennas in ad hoc networks. We elaborate on the problems that they are capable of solving, discuss their limitations, and identify problems that are yet to be completely solved. We begin with a brief discussion of smart antennas and models that are typically used in studies thus far. We then discuss solutions at the medium access control layer that have been proposed for use with such antennas.

\section{SMART ANTENNA BASICS AND MODELS}

In this section, we describe the different kinds of antenna systems and discuss their characteristics in brief. It is not our intent here to describe the signal processing techniques required for tuning antenna patterns or the associated subject matter in electromagnetic and the interested reader is referred to [4] and [5]. As mentioned earlier, our goal in this paper is to primarily look at the networking challenges that arise due to the deployment of such antennas in ad hoc networks.

\subsection{ANTENNAS IN BRIEF}

Omni-directional antennas are those antennas that radiate or receive energy equally well in all directions. Traditionally these antennas have been considered (or implicitly assumed) for studies related to ad hoc networking. Since these antennas dissipate energy in all directions, they impose limitations on the extent to which the wireless spectrum may be re-used in the network. In [6], it was shown that the capacity of an ad hoc network that uses omni-directional antennas is limited.

Smart antennas, naively speaking, have the ability to receive/transmit energy in a particular direction as compared to other directions. The energy dissipated in the directions other than the desired direction can be quelled when transmitting and filtered out while receiving. Smart antennas also null out the interference caused by other transmissions. The antenna is complemented by an adaptive array processor that decides on the amount of power to be used on each antenna element so that the signals combine together to form a specific antenna pattern. The lack of such signal processing techniques causes energy to be dissipated in directions other than the desired one.

An antenna that simply beam forms the energy in a particular direction is often referred to as a directional antenna. Most of the work to date has looked primarily at the use of directional antennas in ad hoc networks. Figure 1.1 depicts the antenna patterns of (a) an omni-directional antenna and (b) a directional antenna. The antenna footprint of a directional antenna contains a main lobe and side labes as shown in the figure. The Yagi antenna [7] is a well known 
directional antenna often used in cellular networks. It has been shown that the capacity of ad hoc networks can be increased significantly by using directional antennas [8].

We further classify directional antennas systems into switched beam (or sectrorized) antenna systems and steer able beam systems. In switched beam systems only multiple fixed beams are possible. As an example, space might be divided into four sectors of $45^{\circ}$ each. A directional transmission would then cover one of these four fixed sectors. A given node in the network cannot focus its antenna beam on a particular neighborhood node so as to maximize the signal strength at that node. Clearly, for a switched beam antenna with $\mathrm{K}$ beams, the width of each beam is $2 \pi / \mathrm{K}$ radians. In contrast, in a steered beam systems, the main lobe of the antenna can be focused in practically any desired direction. Thus, if a given node is communicating with its neighbor, it can adaptively steer its beam so as to point the main lobe towards that neighbor in a mobile scenario as well.

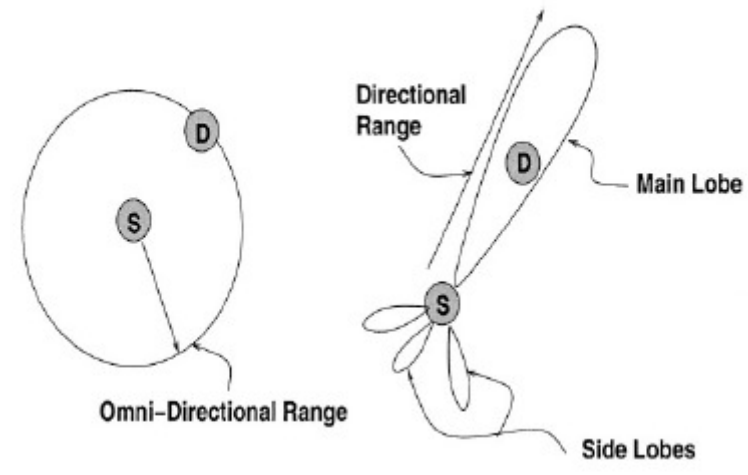

Figure 1.1. Footprint of (a) An Omni-directional Antenna and

(b) A Directional Antenna

The nomenclature smart antennas typically refer to more sophisticated antenna arrays. There are dynamic phased arrays that maximize the gain towards a target in the presence of multi-path effects and there are adaptive arrays that can produce nulls so as to eliminate the effects of simultaneously ongoing interfering transmissions.

We point out that the antennas that we consider for deployment in ad hoc networks are electronically steerable antennas. High-gain aperture and horn antennas that are commonly used in satellite or microwave based terrestrial wireless networks are inappropriate for use in mobile terminals. These antennas will have to be mechanically steered and could be extremely expensive in terms of the energy consumed; this in turn could significantly increase the energy usage in the mobile terminal and could quickly cause its battery to die.

\section{IMPORTANT ANTENNA PARAMETERS}

The gain of a directional antenna is typically higher than that of an omni-directional antenna. Correspondingly directional antennas can have higher reach ability or in other words, a larger directional range as compared to an omni-directional antenna. The gain of a directional antenna is defined as [3]:

$$
\mathrm{G}_{\mathrm{d}}=\frac{{ }_{\mathrm{n}} \mathrm{U}(\mathrm{d})}{\mathrm{U}_{\mathrm{ave}}}
$$


Where, $\mathrm{n}$ is defined to be the efficiency of the antenna and accounts for the hardware related losses, $\mathrm{U}(\mathrm{d})$ is the energy in the direction $\mathrm{d}$ and $\mathrm{U}_{\mathrm{a}} \mathrm{ve}$ is the average power density. The gain is measured in $\mathrm{dBi}$, where $\mathrm{i}$ is used to indicate that this is the gain in decibels over an ideal isotropic antenna.

The main lobe of the antenna represents the direction of peak gain during a transmission or a reception. The antenna beam width typically corresponds to the angle subtended by the two directions on either side of the peak gain that are $3 \mathrm{~dB}$ lower in gain as compared to the peak gain. Note that this is a reduction by half in terms of the signal power as compared to the power in the direction of peak gain (not in decibels). This angle is also sometimes referred to as the $3 \mathrm{~dB}$ beam width.

\section{DIRECTIONAL ANTENNA MODELS}

The presence of side-lobes causes interference to other simultaneous transmissions in spite of using a directional antenna. Sophisticated antenna arrays can steer these side lobes so as to create nulls towards other simultaneous users of the channel. However, simpler (and hence cheaper) antennas suffer from the presence of these side lobes. In ad hoc network literature, most of the work thus far adopts one of two models for characterizing the radiation pattern of a directional antenna: (a) The Flat Topped Radiation Pattern and (b) The Cone sphere Radiation Pattern.

With the flat topped radiation pattern model, it is assumed that the gain of the antenna is a constant within a defined beam width of a radiation. It is also assumed that the side lobes are absent. If the beam width is $\theta$, the gain is computed to be $\mathrm{G}=2 \pi / \theta$.

With the cone and sphere radiation pattern, the side lobes are accounted for by a spherical footprint that is attached to the apex of a cone. The axis of this cone passes through the direction of peak gain of the antenna. If the gain in the direction of the main lobe and the beam width of the main lobe are known, it is a simple exercise to compute the gain of the spherical side-lobe in the cone and sphere radiation pattern [3]. We depict the cone and sphere radiation pattern in Figure 1.2.

\section{MEDIUM ACCESS CONTROL WITH DIRECTIONAL ANTENNAS}

In this section, we describe the current state of the art literature on medium access control with directional antennas. Medium access control refers to the arbitration of the channel bandwidth among a plurality of multiple-access users. We can classify medium access protocols into two types (a) on-demand or unscheduled access and (b) scheduled access. On-demand or unscheduled access mechanisms are based on contention access. Nodes in the ad hoc network contend for the channel. Carrier sensing (both virtual and physical) are used to reduce the extent of packet losses due to collisions. Traditionally, the MAC protocol defined in the IEEE 802.11 standard has been popularly adopted as the Contention based MAC protocol in ad hoc network research. Scheduled access, on the other hand, attempts to schedule transmissions in advance to 


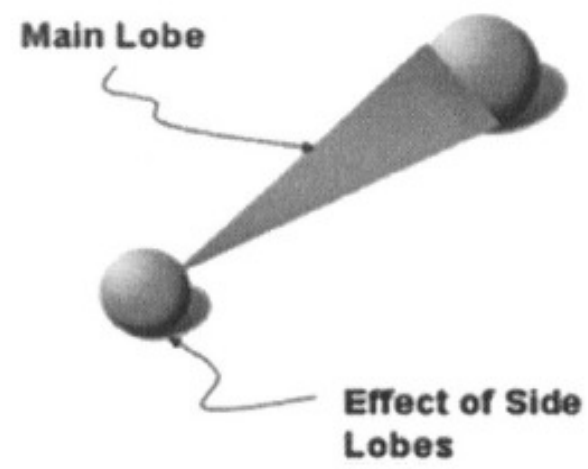

Figure 1.2. The Cone and Sphere Radiation Pattern

reduce the possibility of collisions. Protocols that use scheduled access might proactively allocate bandwidth based on a number of criteria that may include the topology, the generated traffic and priority of various nodes.

We begin with a very brief discussion of the Directional Medium Access Control and the various approaches that have been proposed. Finally, we discuss the few approaches that have been proposed for scheduled access.

\section{DIRECTIONAL MEDIUM ACCESS CONTROL WITH OMNI- DIRECTIONAL RECEPTIONS}

There are two approaches to deal with this problem. The first approach which is called the conservative approach precludes a node from performing transmissions upon the receipt of any control message. The second approach which they call the aggressive approach, allows a node to initiate new transmissions in spite of hearing control messages sent by other nodes. RTS and CTS messages are assumed to be transmitted and received omni-directionally. The RTS and CTS messages are assumed to contain location information of both the sender and receiver; this in turn helps transmit (or receive) the DATA and ACK messages directionally. Neither of the two schemes overcome the problems discussed above. The performance evaluation of the two schemes in [3] shows that both schemes outperform the IEEE 802.11 MAC with omnidirectional communications. The aggressive scheme was found to be better since the conservative scheme suffered from extremely high latencies due to the nodes deferring their communications over extended periods even when the channel was free in reality.

\subsection{SCHEDULED MEDIUM ACCESS CONTROL}

The MAC protocol described thus far are based on collision avoidance. These protocols suffer from high collision rates when the load is high. An alternative approach is to have scheduled access wherein nodes exchange control messages that allow them to know of each other's traffic patterns and thereby somehow schedule collision-free (to the extent feasible) transmissions. In this paper, a new protocol called the Receiver Oriented Multiple Access (ROMA) has been proposed for scheduled access with directional antennas. One other difference in this work as compared with other efforts is that the authors assume the presence of multi-beam antenna arrays (MBAA). The advancement of digital signal processing 
technologies facilitates the use of such arrays. With an MBAA a node can generate multiple beams that allow the node to communicate with more than one of its neighbors. It is assumed that the MBAA can generate upto $\mathrm{K}$ transmit antenna beams. The radiation pattern of an MBAA may be depicted as shown in Figure 1.3. The MBAA also has the ability to anull radiations in unwanted directions.

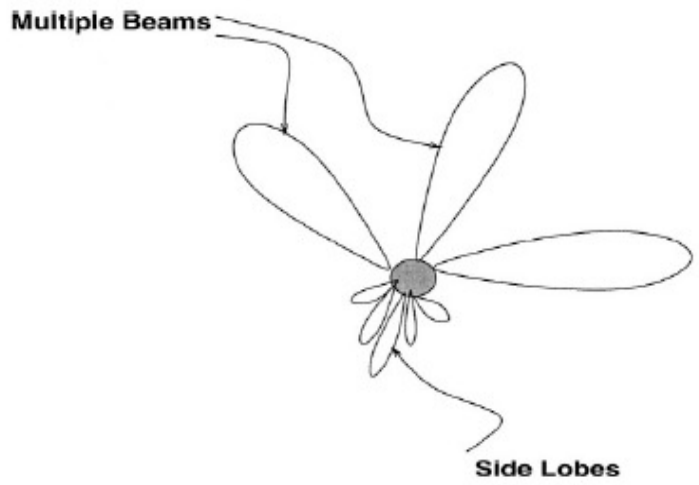

Figure 1.3. The Multi-Beam Antenna Array

We assume that the MBAA system is capable of transmitting to multiple neighbors but is capable of making just a single reception at any given time. Furthermore, they assume the system is capable of performing omni-directional transmissions and receptions. They consider a time-slotted system i.e., time is divided into contiguous frames. The nodes are assumed to have a synchronized view of time by using either the global positioning system (GPS) or the network time protocol (NTP). Each node is assumed to know the precise location of its onehop neighbors.

Each node then propagates its one hop neighbor information to all of its one hop neighbors. Thus, this propagation gives each node knowledge of its two-hop neighborhood. In order to propagate this information, the authors assume that the nodes use omni-directional random access transmissions. Receptions are omni-directional as well. In order to accommodate this, the authors split time into segments. In the scheduled access segment the time is further divided into slots and access in these slots is in accordance to a schedule to be described later. In the random access segment, nodes exchange the control information. In [7] the authors do a simple analysis to compute the fraction of time needed for the random access and show that this is fairly small.

The scheduled access takes the following scenarios into account: (a) avoidance of hidden terminal problems wherein a recipient node ends up receiving transmissions from two simultaneous senders that are hidden from each other (b) ensures that the schedule respects the half-duplex nature of the communications (c) two transmitters are not trying to reach the same receiver at the identifier computes a priority for itself. This priority is based on the use of a simple hash function. Similarly it computes priorities for each of its neighbors. Depending on the traffic generated and its relative priority, a node will make a decision on whether or not to transmit in a particular scheduled slot. Note that the aforementioned scenarios are to be taken into account while this decision is being made. A similar computation is made on the links on which a node would transmit. As a simple example, if a node is of lower priority, it might be unable to transmit on a subset $\mathrm{L}$ of its $\mathrm{K}$ possible links since there are higher priority nodes using those links. 
In addition to this priority assignment, a node will also have to either take the role of a transmitter or a receiver during each slot. If the calculated priority is even, then the node decides to be a receiver and if it is odd, it chooses to transmit. There could be pathological cases wherein a node and all of its neighbors are all either transmitters or receivers. In such a case, the node from the group that has the highest priority will switch its configuration; in other words, if there is a particular group created such that a node and all of its neighbors are receivers, the node with the highest priority in that group will switch to being a receiver. ROMA offers collision free access and has been shown to perform well. However, mobile scenarios are not considered. Furthermore, the priorities based on which the schedules are formed are based on identifiers and not based on the traffic generated.

\section{CONCLUSION}

In the paper we examine the various challenges that arise when deploying smart antennas in ad hoc networks. Most of the protocols designed thus far assume that the antennas are simple directional antennas. The work on medium access control involves using the antennas intelligently for collision avoidance and spatial re-use purposes. Most of the protocols are based on directional virtual carrier sensing wherein overhearing nodes simply preclude transmissions in the direction of an ongoing communication as opposed to not transmitting in any direction as would be the case if a omni-directional antenna were to be used. Although there has been some work on protocols that exploit the use of directional antennas in ad hoc networks, the protocols cannot completely overcome some of the problems that arise due to the use of such antennas and are not yet designed to aptly cope with mobility. Challenges remain and the area remains an exciting and open for future research.

\section{REFERENCES}

[1] A.Acampora and S.V.Krishnamurthy. A new adaptive mac layer protocol for broadband packet Wireless networks in harsh fading and interference environments. IEEE/ACM Transactions on Networking, 2000.

[2] S.V.Krishnamurthy, A.Acampora, and M.Zorzi. Polling based media access protocols for use with smart adaptive array antennas. IEEE/ACM Transactions on Networking, 2001.

[3] R.Ramanathan. On the performance of ad hoc networks with beam forming antennas. Proceedings of ACM MOBIHOC, 2001.

[4] J.C.Liberti and T.S.Rappaport. Prentice Hall, 1999.

[5] J.Kraus and R.J.Marhelka. McGraw Hill, 2002.

[6] P.Gupta and P.R.Kumar. The capacity of wireless networks. IEEE Transactions on Information Theory, 1998.

[7] L.Bao and J.J.Garcia-Luna-Aceves. Transmission scheduling in ad hoc networks with directional antennas. ACM MOBICOM, 2002.

[8] S.Yi, Y.Pei, and S.Kalyanaraman. on the capacity improvement of ad hoc wireless networks using directional antennas. ACM MOBIHOC, 2003. 


\section{Authors}

Mohammed Ali Hussain received the Master's degree M.Sc Computer Science from Alagappa University in 2003. He received Master's degree M.Tech in Information Technology from Allahabad Deemed University in 2005. He received Ph.D. degree in Computer Science from Magadh University, Bihar, India in 2008. He is doing Post Doctoral degree in Computer Science \& Engineering from Acharya Nagarjuna University, A.P., India. He is currently an Assoc. Professor \& Head in the Department of Computer Science \& Engineering in Sri Sai Madhavi Institute of

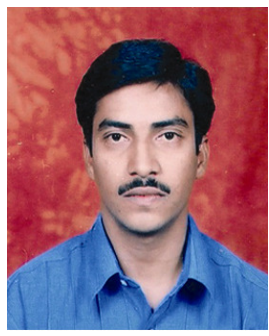
Science \& Technology, Rajahmundry, Andhra Pradesh, India. He had published several papers in National and International Conferences \& International Journals. His research interests are Wireless Networks with specialization in Quality of Service (QoS) in IEEE 802.11 Wireless LANs. \& Ad-Hoc Networks. He is a member of IACSIT and ISTE.

P.Suresh Varma received the Master's degree M.Tech in Computer Science \& Technology from Andhra University in 1998. He received Ph.D. degree in Computer Science \& Engineering from Acharya Nagarjuna University in 2008. He is currently an Professor in Department of Computer Science \& Engineering in Adikavi Nannaya University, Rajahmundry, A.P., India. He had published several papers in National and International Journals. He is active member of various professional bodies.

K. Satya Rajesh received the Master's degree M.C.A from Manonmaniam Sundarnar University in 2003. He received Master's degree M.Tech in Computer Science \& Engineering from Acharya Nagarjuna University in 2010. He is doing Ph.D. in Computer Science from Rayalaseema University, Kurnool, A.P., India. He is currently an Asst.Professor in Department of Computer Science \& Engineering in Vikas College of Engineering \& Technology, Vijayawada, A.P., India. His research interests are Computer Networks \& Sensor Networks.

Hussain Basha Pathan received the Master's degree M.C.A from Acharya Nagarjuna University in 2007. He received Master's degree M.Tech in Computer Science \& Engineering from Acharya Nagarjuna University in 2010. He is doing Ph.D. in Computer Science from Rayalaseema University, Kurnool, A.P., India. $\mathrm{He}$ is currently an Asst.Professor in Department of M.C.A in S.N.B.T.M (PG) College, Repalle, Guntur Dist., A.P., India. His research interests are Wireless and Ad-hoc Networks.

Leela Madhav Sarraju received the Master's degree M.C.A from Acharya Nagarjuna University in 2000. He received Master of Philosophy (M.Phil) in Computer Science from Periyar University in 2008. He is doing Ph.D. in Computer Science from Dravidian University, Kuppam, A.P., India. His research interests are Wireless and Ad-hoc Networks. He is a member of CSI \& IEEE.
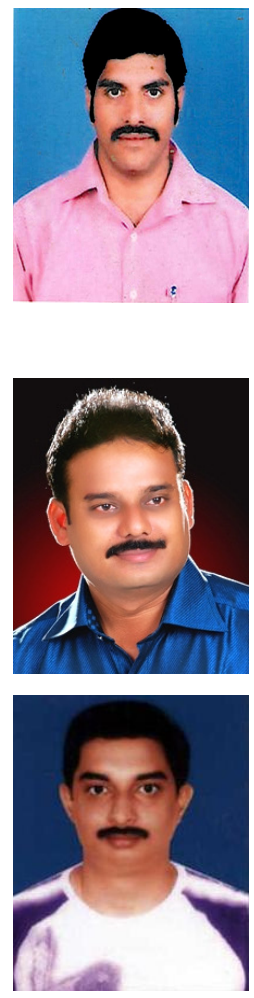